\title{
Effects of combined UV and chlorine treatment on chloroform formation from triclosan
}

\author{
Weiwei Ben ${ }^{\text {a, b }}$, Peizhe Sun ${ }^{\text {b }}$, Ching-Hua Huang ${ }^{\text {b, * }}$ \\ ${ }^{a}$ Research Center for Eco-Environmental Sciences, Chinese Academy of Sciences, Beijing 100085, People's Republic of China \\ ${ }^{\mathrm{b}}$ School of Civil and Environmental Engineering, Georgia Institute of Technology, Atlanta 30332, GA, United States
}

\section{H I G H L I G H T S}

- The combined UV/chlorine notably enhances the chloroform formation from triclosan.

- UV photolysis plays an important role in the enhancement of chloroform formation.

- Chlorinated triclosan intermediates act as important precursors of chloroform.

- The promotion of DBPs needs to be considered in the combined UV/ chlorine treatment.

\section{A R T I C L E I N F O}

\section{Article history:}

Received 31 October 2015

Received in revised form

16 December 2015

Accepted 19 December 2015

Available online 30 December 2015

Handling Editor: J. de Boer

Keywords:

Chlorination

UV treatment

Triclosan

Chloroform

Disinfection by-products
G R A P H I C A L A B S T R A C T

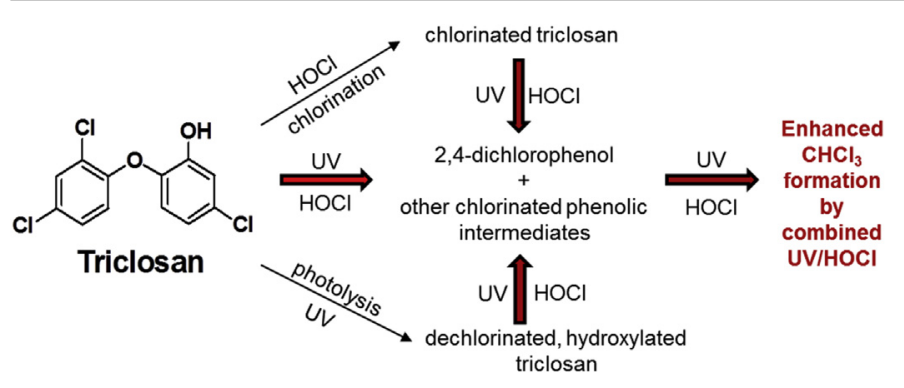

\begin{abstract}
A B S T R A C T
The co-exposure to UV irradiation and free chlorine may occur in certain drinking water and wastewater treatment systems. This study investigated the effects of simultaneous low pressure ultraviolet (LPUV) irradiation and free chlorination on the formation of chloroform from triclosan which is a commonly used antibacterial agent. Different treatment systems (i.e., combined UV/chlorine, UV alone, and chlorine alone) were applied to examine the degradation of triclosan and formation of chloroform. The fate of representative intermediates, including chlorinated triclosan, dechlorinated triclosan intermediates and 2,4-dichlorophenol, were tracked to deduce the effect of combined UV/chlorine on the transformation of chloroform formation precursors. The relation between intermediates degradation and chloroform formation was investigated in depth by conducting stepwise experiments with UV and chlorine in different sequences. Results indicate that the combined UV/chlorine notably enhanced the chloroform formation from triclosan. From the reaction mechanism perspective the combined UV/chlorine, where the direct photolysis may play an important role, could accelerate the decay of intermediates and facilitate the generation of productive chloroform precursors. The radicals had modest influence on the degradation of triclosan and intermediates and partly hindered the formation of chloroform. These results emphasize the necessity of considering disinfection by-products formation in the application of combined UV/ chlorine technology during water treatment.
\end{abstract}

() 2015 Elsevier Ltd. All rights reserved.

\footnotetext{
* Corresponding author.

E-mail address: ching-hua.huang@ce.gatech.edu (C.-H. Huang).
}

\section{Introduction}

UV disinfection is receiving increased attention, particularly from those who seek to reduce concentrations of disinfection byproducts (DBPs). Due to UV disinfection's inability to provide 
residual protection, UV is often used in conjunction with chlorine disinfectants in a complementary manner. In many UV disinfection installations, free chlorine is present in the water as it passes through the UV reactors (Feng et al., 2007). The co-exposure to UV irradiation and free chlorine may be intentional or unintentional. For example, in the rooftop water storage tanks in some areas equipped with small-scale UV units as a final water purification step, the photolysis of chlorine may occur (Wait, 2008). In some cases, the residual chlorine could be exposed to UV photolysis (sunlight), like in outdoor swimming pools and reservoirs.

The potential of simultaneous UV irradiation and chlorination (referred to as combined UV/chlorine hereafter) as an advanced oxidation process (AOP) has been explored by various studies due to the formation of $\mathrm{OH}$ radicals from the photolysis of free chlorine (Nowell and Hoigné, 1992; Jin et al., 2011; Fang et al., 2014). Hence, the UV-driven chlorine process can be used to destroy hazardous organic compounds in drinking water and wastewater. Jin et al. (2011) confirmed the $\mathrm{OH}$ radical production in the combined UV/ chlorine process. Sichel et al. (2011) determined the feasibility of the UV/chlorine AOP for removal of various emerging contaminants. In that work, UV/chlorine turned out to be a lower energy AOP compared to the $\mathrm{UV} / \mathrm{H}_{2} \mathrm{O}_{2}$ process, yielding energy reduction by $30-75 \%$ for several environmentally relevant emerging contaminants. Previous studies have indicated that UV irradiation combined with chlorination (in sequence or simultaneously) may affect the reaction processes and thus the generation of products. The UV irradiation at $254 \mathrm{~nm}\left(U_{2} V_{24}\right)$ promoted several reactions that were involved in the formation and/or destruction of chlorinated N-DBPs in swimming pools in the presence of chlorine or monochloramine (Weng et al., 2012; Soltermann et al., 2013). Zhao et al. (2011) found that medium-pressure UV and chlorine coexposure of nitrobenzene and benzoic acid enhanced the formation of total organic halogen. Our previous study showed that trichloronitromethane (TCNM) formation could increase up to 15 fold by combined $\mathrm{UV}_{254} /$ chlorine under disinfection to advanced oxidation conditions, owing to that the combined $\mathrm{UV}_{254} /$ chlorine conditions significantly promoted the generation of better TCNM precursors and facilitated their transformation to TCNM (Deng et al., 2014).

Triclosan (5-chloro-2-(2,4-dichlorophenoxy) phenol), owing to its antibacterial, antifungal and antiviral properties, is commonly used in many personal hygiene products such as hand soaps, deodorants and toothpastes at concentrations ranging from 0.1 to $1 \%$ by weight (Rule et al., 2005). The consumption of triclosan in this vast array of daily necessities causes its migration in the environment after it is washed down the drain. However, it was observed that a considerable portion of triclosan in wastewater may survive treatment of wastewater treatment plants (WWTPs) and then discharge with water or sit in sewage sludge (Lindström et al., 2002; Singer et al., 2002; Heidler and Halden, 2007). Triclosan was detected at concentrations of $23-434 \mathrm{ng} \mathrm{L}^{-1}$ in nineteen effluents from Australian WWTPs and up to $75 \mathrm{ng} \mathrm{L}^{-1}$ in surface waters from five rivers receiving effluent discharge from WWTPs (Ying and Kookana, 2007). In the United States, triclosan was one of the most frequently detected micropollutants, with detection frequency of 57.6\% in 139 tested U.S. streams and rivers (Kolpin et al., 2002). In samples collected from four water filtration plants located in southern California, triclosan was detected at the total mean concentration of $56-49 \mathrm{ng} \mathrm{L}^{-1}$ in raw drinking water and finished drinking water (before chlorination) (Loraine and Pettigrove, 2006). Although triclosan was usually detected at low concentrations, chronic, low-level exposure for multiple generations may still cause ecosystem problems by interfering with species low on the food chain. Thus, due to the concerns about potential human toxicity and the antibiotic resistance development, triclosan is currently the focus of several regulatory efforts and a ban on triclosan-containing soaps and cleaning products has passed by legislators in Minnesota (Kemsley, 2014).

In water disinfection processes, triclosan can react quickly with free chlorine to produce chlorinated products including chlorinated phenoxy-phenols and chlorinated phenols, and finally results in formation of chloroform (Rule et al., 2005; Fiss et al., 2007). Chloroform is a well-known DBP in the chlorine disinfection process and has been classified by the U.S. Environmental Protection Agency (USEPA) as a probable human carcinogen. On the other hand, UV photolysis could be a promising method to degrade triclosan without generation of chlorinated triclosan derivatives (Buth et al., 2011). Son et al. (2007) found that triclosan photodegradation followed the pseudo-first order kinetics at the wavelength of $254 \mathrm{~nm}$ and the photodegradation rate was $1.32 \mathrm{~min}^{-1}$ at the UV intensity of $1.08 \times 10^{4} \mathrm{E} \cdot \mathrm{L}^{-1} \cdot \mathrm{min}^{-1}$. To our best knowledge, no study has thus far analyzed the degradation rates and products formation of triclosan under the combined UV/chlorine treatment system.

In this study, bench-scale experiments using a low pressure ultraviolet (LPUV) lamp were conducted to investigate the efficiency of the combined LPUV/chlorine treatment for removing triclosan and characterize the effects on the fate of triclosan reaction intermediates and the formation of chloroform by-product. On the basis of the experimental results, reaction pathways of triclosan degradation and chloroform formation under combined UV/chlorine treatment were proposed.

\section{Materials and methods}

\subsection{Chemical reagents}

Triclosan ( $\geq 97 \%$ ), 2,4-dichlorophenol (99\%) and chloroform (99.9\%) were purchased from Sigma-Aldrich. Methyl tertiary-butyl ether (MTBE), methanol and acetonitrile of HPLC grade were obtained from Fisher Scientific. All other reagents used (e.g., buffers, colorimetric agents, reductants, etc.) were obtained from Fisher Scientific and were of at least reagent grade quality. Deionized (DI) water, from a Milli-Q Ultrapure Gradient A10 (Millipore-Billerica, MA) water purification system with a resistivity of $>18 \mathrm{M} \Omega$, was used to prepare all reagent solutions. Free chlorine stock solutions was prepared by diluting the $\mathrm{NaOCl}$ solution ( $7 \%$ solution concentration, obtained from Fisher Scientific) with DI water and standardized by iodometry and $N, N$-diethyl-p-phenylenediamine ferrous ammonium sulfate (DPD-FAS) titrimetry (APHA, 1998). Triclosan stock solution of $1 \mathrm{~g} \mathrm{~L}^{-1}$ was prepared in water by adding $\mathrm{NaOH}$. 2,4-Dicholophenol stock solution was prepared in DI water at $1 \mathrm{~g} \mathrm{~L}^{-1}$. Chloroform stock solution of $20 \mathrm{mg} \mathrm{L}^{-1}$ was prepared by diluting the standard with DI water. Phosphate buffer ( $\mathrm{pH} 7.0$ ) of $10 \mathrm{mM}$ was used to maintain constant $\mathrm{pH}$ during experiments.

\subsection{Experimental setup}

All experiments were conducted in a magnetically stirred 100$\mathrm{mL}$ cylindrical quartz reactor. For triclosan degradation experiments, $85 \mathrm{~mL}$ of sample with $0.02 \mathrm{mM}$ of triclosan in phosphate buffer was first placed into the quartz reactor. Then the quartz reactor was placed into a photochamber similar to that in Deng et al. (2014). A certain volume of free chlorine stock solution was added into the reactor through the circular hole in the photochamber's top to start the reaction, with selected initial free chlorine/triclosan (FAC:T) ratios of 5:1, 2:1 and 1:1, respectively. UV treatment was supplied by a 4-W LPUV lamp (G4T5 Hg lamp, Philips TUV4W) peaking at $254 \mathrm{~nm}$ at ambient temperature $\left(25^{\circ} \mathrm{C}\right)$. The incident light intensity was $2.0 \mathrm{~mW} \mathrm{~cm}^{-2}$ to the reactor 
measured by a UVX radiometer. The lamp was warmed up for at least 30 min prior to the experiments. A series of $1 \mathrm{~mL}$ of samples were taken out at different reaction times, and the residual chlorine was quenched immediately with excess sodium thiosulfate. For examining the possible influence of $\mathrm{OH}$ radical, $10 \mathrm{mM}$ tert-butanol (TBA) was added before free chlorine. Experiments to determine the transformation from 2,4-dichlorophenol to chloroform were conducted by the same procedures as triclosan degradation, with the initial concentrations of 2,4-dichlorophenol and free chlorine at $0.02 \mathrm{mM}$ and $0.1 \mathrm{mM}$, respectively. All experiments were conducted in duplicate, and the relative standard deviations of all data points were determined to be less than $10 \%$.

\subsection{Chemical analysis}

Triclosan, chlorinated triclosan intermediates (4-Cl-triclsoan, 6Cl-triclosan and 4,6-Cl-triclosan) and dechlorinated triclosan intermediates were monitored using an Agilent HPLC-MS (1100 HPLC/ G1956B MSD) system with a Zorbax SB-C18 column $(2.4 \times 150 \mathrm{~mm}$, $5 \mu \mathrm{m})$. The injection volume of each sample was $20 \mu \mathrm{L}$ and the column temperature was $40^{\circ} \mathrm{C}$. A binary gradient of 18 min with a flow rate of $0.25 \mathrm{~mL} \mathrm{~min}^{-1}$ was adopted. Mobile phase A was $0.1 \%$ acetic acid and $0.1 \%$ ammonium acetate aqueous solution $(\mathrm{v} / \mathrm{v})$. Mobile phase $B$ was a mixture of acetonitrile and methanol $(1: 1, v / v)$. The gradient program was as the following: $40 \% \mathrm{~B}$ from 0 to $3 \mathrm{~min}, 100 \% \mathrm{~B}$ from 9.5 to $12.5 \mathrm{~min}$, and $40 \%$ B from 13 to $18 \mathrm{~min}$; the mobile phase ratio was changed linearly for each ramp. Electrospray ionization at negative mode $\left(\mathrm{ESI}^{-}\right)$was applied with fragmentor voltage of $220 \mathrm{~V}$ and mass scan range of $m / z 50-500$. The drying gas was at $6 \mathrm{~L} \mathrm{~min}^{-1}$ at $350{ }^{\circ} \mathrm{C}$, the nebulizer pressure $25 \mathrm{psig}$, and the capillary voltage 4000 V. Triclosan, 4-Cl-triclsoan \& 6-Cl-triclosan, 4,6-Cl-triclosan and dechlorinated triclosan intermediates (resulting from one chlorine loss of triclosan) were identified on the basis of their elution time and molecular ions at $m / z 287 / 289 / 291,321 / 323 / 325,355 / 357 /$ 359 and 253/255, respectively. An example of the extracted-ion chromatograms of triclosan and the tested intermediates are shown in the Supporting information Fig. S1, which was a combined UV/chlorine reaction system with initial FAC:T of 2:1. Only triclosan was quantified because of the absence of analytical standards for the other chlorinated/dechlorinated triclosan intermediates. Calibration standards of triclosan in the range of $0.1-5 \mathrm{mg} \mathrm{L}^{-1}$ yielded a good linear correlation $\left(\mathrm{R}^{2}=0.998\right)$. The limit of quantification (LOQ) for triclosan was $5.0 \mu \mathrm{g} \mathrm{L}^{-1}$ in the test samples.

Chloroform and 2,4-dichlorophenol were quantified using a liquid-liquid extraction (LLE) procedure according to the USEPA Method 551. After the LLE procedure, about $0.8 \mathrm{~mL}$ of extract in MTBE was obtained and then analyzed using an Agilent gas chromatograph equipped with an Agilent DB-5 column $(0.25 \mathrm{~mm}$ ID $\times 30 \mathrm{~m})$ and an electron capture detector $(\mathrm{ECD})$. The GC conditions were as the following: the initial oven temperature was $35^{\circ} \mathrm{C}$ and was held for $4 \mathrm{~min}$; the temperature was then increased to $120^{\circ} \mathrm{C}$ at a rate of $15^{\circ} \mathrm{C} \mathrm{min}^{-1}$ and was held for $2 \mathrm{~min}$; the injector temperature was set at $200{ }^{\circ} \mathrm{C}$ and the detector temperature at $250^{\circ} \mathrm{C}$. Calibration curves of 2,4-dichlorophenol and chloroform were conducted using extracts of serial dilutions of analytical standard. Good linear correlations $\left(R^{2}=0.997-0.999\right)$ were achieved in the range of $0.1-5 \mathrm{mg} \mathrm{L}^{-1}$ for both 2,4 -dichlorophenol and chloroform. The LOQs for chloroform and 2,4-dichlorophenol were 0.4 and $1.2 \mu \mathrm{g} \mathrm{L}^{-1}$, respectively.

\section{Results and discussion}

\subsection{Degradation of triclosan under combined UV/chlorine}

To evaluate the effect of combined UV/chlorine on the degradation of triclosan, a series of batch experiments were conducted with a range of initial FAC:T molar ratios and LPUV irradiation intensity of $2.0 \mathrm{~mW} \mathrm{~cm} \mathrm{~cm}^{-2}$. As shown in Fig. 1, with free chlorine only, the higher the initial free chlorine concentration was, the faster triclosan degraded. Triclosan could be completely degraded within 3 min with the FAC:T ratio of $5: 1$ (i.e., $[\mathrm{FAC}]_{0}=7.1 \mathrm{mg} \mathrm{Cl}_{2} \mathrm{~L}^{-1}$ ). With the lower FAC:T ratios of $2: 1$ and $1: 1$ (i.e., $[\mathrm{FAC}]_{0}=2.8$ and $1.4 \mathrm{mg} \mathrm{Cl}_{2} \mathrm{~L}^{-1}$ ), the degradation of triclosan showed fast rates within $5 \mathrm{~min}$, then slowed down from $5 \mathrm{~min}$ to $60 \mathrm{~min}$, and finally reached residual triclosan concentrations of 0.76 and $7.69 \mu \mathrm{M}$, respectively. The combined UV/chlorine enhanced the degradation of triclosan compared to free chlorine only, with the triclosan degradation rate increased slightly at the FAC:T ratio from $2: 1$ to $5: 1$.With the FAC:T ratio of $5: 1$, the UV irradiation caused rapid degradation of triclosan in the initial $2 \mathrm{~min}$, and led to more than $98 \%$ removal of triclosan at $5 \mathrm{~min}$. The pseudo-first order approximation was applied to simulate the degradation kinetics of triclosan within 2 min and the determined pseudo-first-order rate constants $\left(k, \mathrm{~min}^{-1}\right)$ are listed in Table S1. With free chlorine only, the $k$ values were determined as $1.56 \pm 0.12,0.27 \pm 0.02$ and $0.09 \pm 0.01$ with the FAC:T ratios of 5:1, $2: 1$ and $1: 1$, respectively, whereas under the combined $\mathrm{UV} /$ chlorine conditions the $k$ values increased to $1.93 \pm 0.07,1.28 \pm 0.04$ and $0.75 \pm 0.01$ at corresponding FAC:T ratios, respectively. These results indicate that the impact of FAC:T ratio on triclosan degradation was diminished under the combined process and the UV photolysis might play an important role in the degradation of triclosan. Indeed, the fact that degradation rate of triclosan with UV irradiation only $\left(k=0.37 \pm 0.03 \mathrm{~min}^{-1}\right)$ exceeded that with free chlorine only at FAC:T of 2:1 and 1:1 also emphasized the contribution of UV photolysis. The addition of excess amount of TBA $(\bullet \mathrm{OH}$ and $\bullet \mathrm{Cl}$ scavenger) exhibited little influence on the degradation of triclosan.

\subsection{Chloroform formation from triclosan under combined UV/ chlorine}

Fig. 2 shows the molar yields of chloroform from triclosan under different treatment processes. With free chlorine only, chloroform was formed continuously within the reaction time of $60 \mathrm{~min}$ and the molar yields of chloroform reached 0.13 and 0.03 at FAC:T ratios

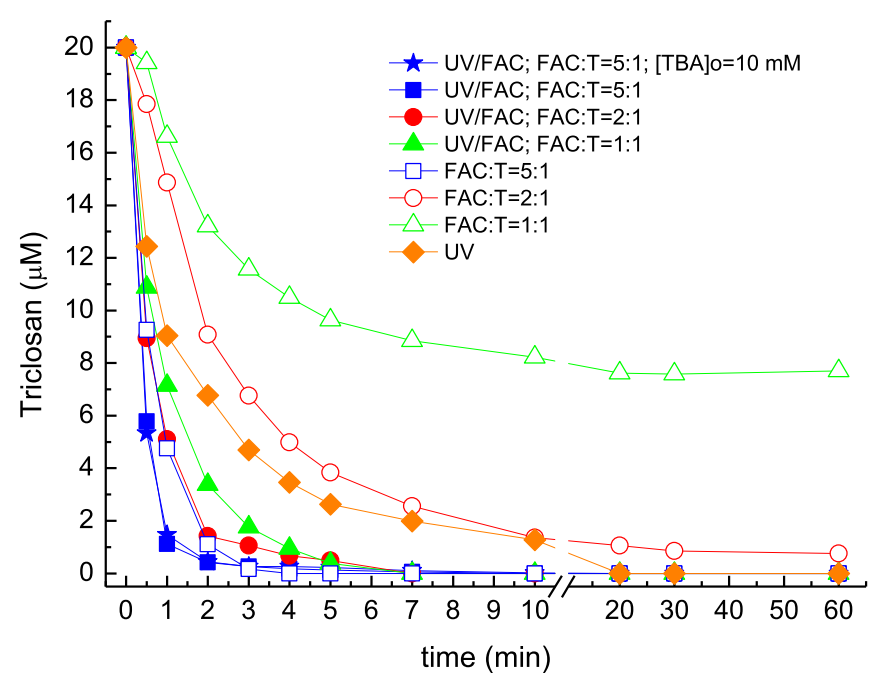

Fig. 1. Degradation of triclosan under combined UV/chlorine, UV irradiation alone and chlorination alone treatment, respectively. Reaction conditions: [triclosan $]_{\mathrm{o}}=0.02 \mathrm{mM}, \mathrm{LPUV}=2.0 \mathrm{~mW} \mathrm{~cm}{ }^{-2}, \mathrm{pH}=7.0, \mathrm{~T}=25^{\circ} \mathrm{C}$; FAC:T represents the initial free chlorine/triclosan molar ratio. 


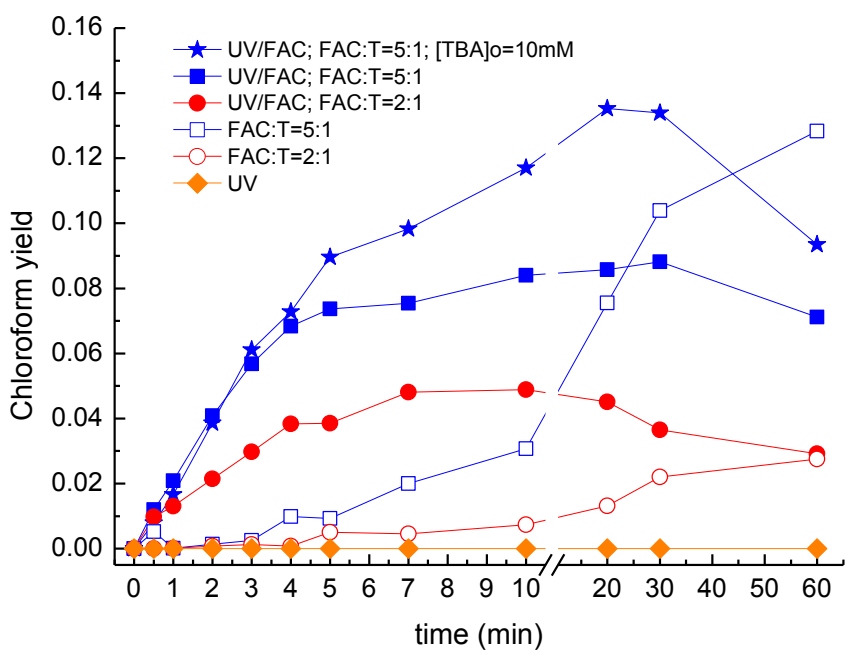

Fig. 2. Molar yields of chloroform from triclosan degradation under combined UV/ chlorine, UV irradiation alone and chlorination alone treatment, respectively. Reaction conditions: [triclosan $]_{\mathrm{o}}=0.02 \mathrm{mM}, \mathrm{LPUV}=2.0 \mathrm{~mW} \mathrm{~cm}^{-2}, \mathrm{pH}=7.0, \mathrm{~T}=25^{\circ} \mathrm{C}$.

of 5:1 and 2:1, respectively. Under combined UV/chlorine at FAC:T ratios of $5: 1$ and $2: 1$, chloroform formation rose quickly to the molar yields of 0.07 and 0.04 at 5 min, respectively, about $83 \%$ and $79 \%$ of the corresponding maximum chloroform yield (i.e., 0.09 and 0.05 at $30 \mathrm{~min}$ and $10 \mathrm{~min}$, respectively) observed. Then the chloroform formation leveled off in the following reaction time and finally decreased to 0.07 and 0.03 at $60 \mathrm{~min}$, respectively, corresponding to $19 \%$ and $40 \%$ decrease from the maximum yields.

The change of free chlorine concentrations under the different experimental conditions was monitored and shown in Fig. S2. Under combined UV/chlorine and the FAC:T ratio of 5:1, free chlorine concentration declined over time to a negligible level at $30 \mathrm{~min}$. It can be predicted that free chlorine was likely depleted sooner than when the FAC:T ratio of 2:1 was employed in the combined UV/chlorine system. Fig. 2 shows that, in the combined UV/chlorine system, chloroform formation was decreased after 10 min at FAC:T ratio of 2:1 whereas the decrease occurred after $30 \mathrm{~min}$ at FAC:T ratio of 5:1. These results indicate the presence of excess free chlorine was important for the formation of chloroform. Without UV irradiation, the FAC:T ratio of 5:1 led to a residual free chlorine concentration of $1.87 \mathrm{mg} \mathrm{L}^{-1}$ at $30 \mathrm{~min}$, which caused the continuous formation of chloroform beyond $30 \mathrm{~min}$ in the chlorination alone treatment system. Overall, the strong dependence of chloroform formation yield on the free chlorine concentration indicates the amount of free chlorine is crucial for the chloroform formation from triclosan under both combined UV/chlorine and chlorination alone processes. Fig. 2 shows that with the addition of TBA, the molar yield of chloroform reached its maximum value of 0.13 at $20 \mathrm{~min}$, which was increased by $44 \%$ compared to that without TBA addition.

\subsection{Fate of reaction intermediates under combined UV/chlorine}

Previous studies have reported several degradation intermediates from chlorination or photolysis of triclosan. Rule et al. (2005) identified three chlorophenoxyphenols and two chlorophenols in the reaction of triclosan with free chlorine. Ferrer et al. (2004) proposed the replacement of chlorine atoms by hydroxyl groups and chlorine losses as the major photolytic degradation pathways of triclosan, and 2,4-dichlorophenol could also be formed from photoinduced hydrolysis of triclosan through cleavage of the $\mathrm{C}-\mathrm{O}$ ether bond. In this work, several degradation intermediates, including three chlorinated triclosan intermediates (i.e., 4-Cl-triclosan, 6-Cl-triclosan and 4,6-Cl-triclosan), one chlorophenol (i.e., 2,4-dichlorophenol) and dechlorinated triclosan intermediates resulting from one chlorine loss of triclosan, were determined in different treatment processes. The analytical standards were unavailable for chlorinated and dechlorinated triclosan, and synthesis and purification of these intermediates were difficult to achieve during the time of the experiments. Therefore, the peak area of corresponding intermediate peak in the LC-MS chromatogram was integrated and applied to present the formation and decay of these intermediates. Since 4-Cl-triclosan and 6-Cl-triclosan were formed or decayed simultaneously, they were not distinguish from each other but summed up by their peak areas instead. In addition, the one chlorine atom loss may occur at any of the three chlorine atoms in triclosan and result in coterminous peaks in the LC-MS chromatogram. Hence, their peak areas were combined to present dechlorinated triclosan.

With free chlorine only, monochlorinated triclosan intermediates were formed quickly and then, at the higher FAC:T ratios, decreased in further reaction time (Fig. 3a). 4,6-Cl-Triclosan was continuously produced and followed by subsequent decay at the higher FAC:T ratio of 5:1 (Fig. 3b). Take the FAC:T of 5:1 for example, triclosan concentration was decreased to a negligible level at around 3 min (Fig. 1) while the corresponding chloroform molar yield was lower than 0.01 (Fig. 2). However, chloroform formation increased significantly after 5 min (Fig. 2) that corresponded to the beginning of the degradation of 4,6-Cl-triclosan (Fig. 3b), suggesting the further degradation products from 4,6-Cltriclosan, rather than triclosan, may act as important precursors of chloroform formation.

With combined UV/chlorine, UV irradiation enhanced both the degradation of chlorinated triclosan intermediates (Fig. 3) and the formation of chloroform (Fig. 2). The degradation of chlorinated triclosan highly correlated with the formation of chloroform. In the initial $5 \mathrm{~min}$, the small peak areas of chlorinated triclosan intermediates may suggest faster degradation of these intermediates, leading to faster formation of chloroform.

Dechlorinated triclosan intermediates were generated quickly under UV irradiation only and combined UV/chlorine, and they also decayed during further reaction time (Fig. 3c). The formation of dechlorinated triclosan intermediates may be partly responsible for the faster degradation of triclosan under combined UV/chlorine than free chlorine only. It is hypothesized that products from the degradation of dechlorinated triclosan intermediates may also act as important precursors of chloroform under combined UV/chlorine treatment.

Fig. 3d shows the evolution of the 2,4-dichlorophenol intermediate from different treatment of triclosan. The accumulated 2,4-dichlorophenol concentration was higher under chlorination only. Under combined UV/chlorine, 2,4-dichlorophenol's generation in the initial $3 \mathrm{~min}$ and subsequent degradation rate in the later time were both enhanced. For comparison, separate experiments were conducted to assess 2,4-dichlorophenol's fate and generation of chloroform under combined UV/chlorine, UV irradiation alone, and chlorination alone. As shown in Fig. S3, the degradation of 2,4dichlorophenol was fastest under combined UV/chlorine and greater than the sum of removal efficiencies caused by the UV irradiation alone and chlorination alone. Correspondingly, the combined UV/chlorine also exhibited a notably higher molar yield of chloroform. This result suggests that combined UV/chlorine can enhance degradation of the phenol intermediates and help produce precursors which are easier to form chloroform (e.g., facilitate the cleavage of intermediates).

The effects of UV irradiation and chlorination on the behavior of intermediates and chloroform formation were further evaluated by 


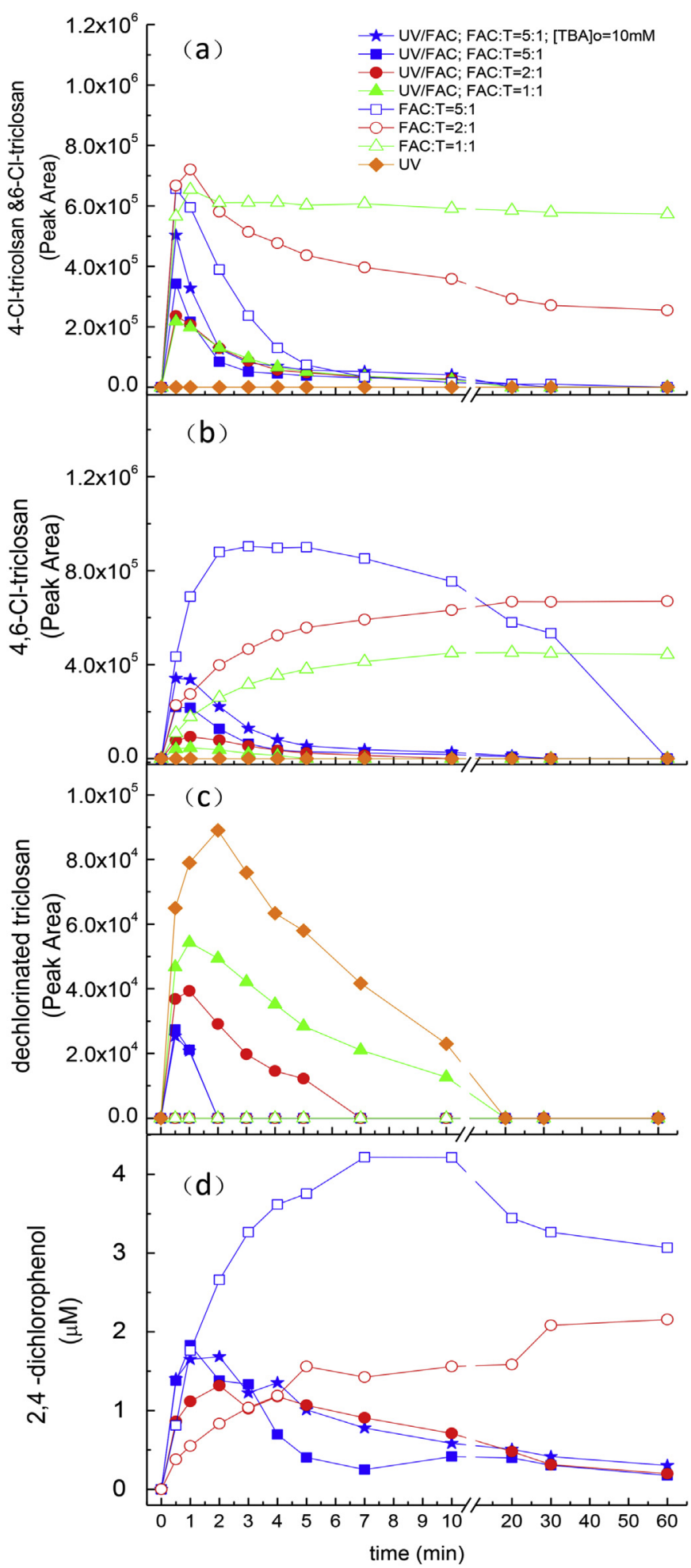

Fig. 3. Fate of intermediates from triclosan degradation under combined UV/chlorine, UV irradiation alone and chlorination alone treatment, respectively: (a) 4-Cl-triclosan \& 6-Cl-triclosan, (b) 4,6-Cl-triclosan, (c) dechlorinated triclosan, (d) 2,4dichlorophenol. Reaction conditions: [triclosan $]_{\mathrm{o}}=0.02 \mathrm{mM}$, LPUV $=2.0 \mathrm{~mW} \mathrm{~cm}^{-2}$, $\mathrm{pH}=7.0, \mathrm{~T}=25^{\circ} \mathrm{C}$.

stepwise experiments with UV and chlorine in different sequences and conditions (chlorination with post-UV exposure or UV exposure with post-chlorination), as illustrated in Fig. 4 and Fig. 5. Under chlorination with FAC:T of 5:1, triclosan could be totally degraded at

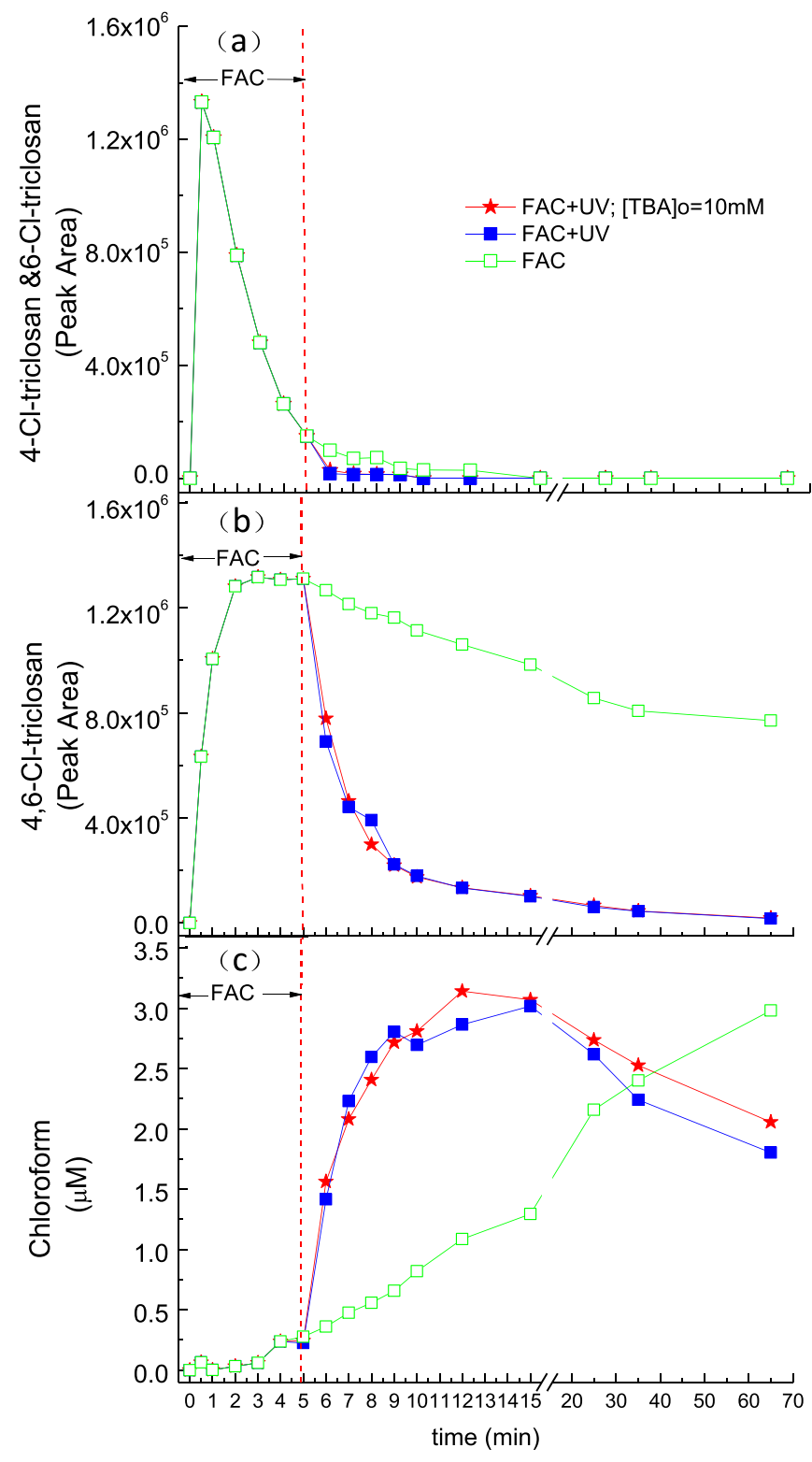

Fig. 4. Time-course behavior of intermediates and chloroform formation subjected to chlorination for 5 min followed by with/without UV irradiation: (a) 4-Cl-triclosan \& 6Cl-triclosan, (b) 4,6-Cl-triclosan, (c) chloroform formation. Reaction conditions: [triclosan $]_{o}=0.02 \mathrm{mM}, \mathrm{FAC}: \mathrm{T}=5: 1, \mathrm{LPUV}=2.0 \mathrm{~mW} \mathrm{~cm}^{-2}, \mathrm{pH}=7.0, \mathrm{~T}=25^{\circ} \mathrm{C}$.

5 min (Fig. 1) and meanwhile about $4.2 \mathrm{mg} \mathrm{L}^{-1}$ of residual free chlorine was detected. Fig. $4 \mathrm{a}$ and $\mathrm{b}$ shows that compared to free chlorine only, combined UV/chlorine significantly enhanced the degradation of chlorinated triclosan intermediates, which facilitated the transformation of these intermediates to chloroform formation precursors (Fig. 4c). The addition of excess TBA slightly enhanced the formation of chloroform (Fig. 4c).

In the other series of experiments, triclosan was first exposed to UV irradiation for $5 \mathrm{~min}$ and that led to around $2.63 \mu \mathrm{M}$ of residual triclosan remaining. Then the solution was subjected to various subsequent reaction conditions. Fig. 5a and Fig. 5b show that the residual triclosan transformed to chlorinated triclosan intermediates when free chlorine was added; however, the low peak areas of chlorinated triclosan indicated that most triclosan had transformed to photolysis products. Fig. 5c shows that the degradation of dechlorinated triclosan intermediates was facilitated by 


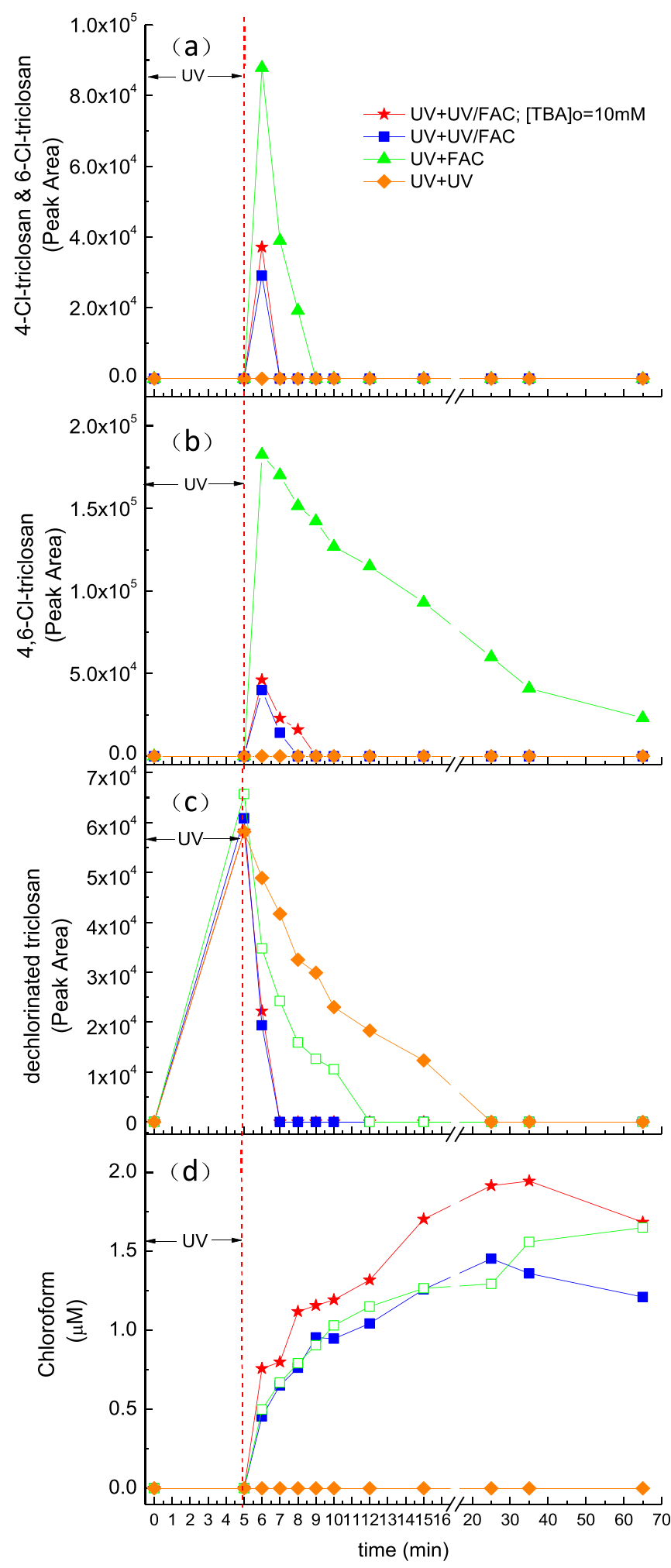

Fig. 5. Time-course behavior of intermediates and chloroform formation subjected to UV irradiation for 5 min followed by with/without chlorination: (a) 4-Cl-triclosan \& 6Cl-triclosan, (b) 4,6-Cl-triclosan, (c) dechlorinated triclosan, (d) chloroform formation. Reaction conditions: [triclosan] $]_{\mathrm{o}}=0.02 \mathrm{mM}, \mathrm{FAC}: \mathrm{T}=5: 1, \mathrm{LPUV}=2.0 \mathrm{~mW} \mathrm{~cm}{ }^{-2}$, $\mathrm{pH}=7.0, \mathrm{~T}=25^{\circ} \mathrm{C}$.

free chlorine as well as UV irradiation. The formation rate of chloroform with post-chlorination was similar to that with postcombined UV/chlorine (Fig. 5d), suggesting that the UV photolysis for 5 min may lead to transformation of triclosan to some intermediates which can easily transform to chloroform in the presence of free chlorine.

\subsection{Proposed pathways of triclosan degradation and chloroform formation by combined UV/chlorine}

Results of this study clearly showed that the combined UV/ chlorine can notably enhance the formation of chloroform from triclosan compared to chlorination alone, especially in the first 10 min under the employed experimental conditions.

Fig. 6a shows the reaction mechanism of triclosan with chlorination alone. Chlorine substitution on the aromatic rings of triclosan dominates and then a series of chlorinated triclosan are generated. Chlorine as an oxidant may lead to opening of triclosan's chlorinated phenol ring that leads to chlorinated ketone intermediates and eventually releasing chloroform after hydrolysis of chlorinated ketones (Arnold et al., 2008). Chlorine may also lead to the ether bond cleavage on triclosan and generate 2,4dichlorophenol. However, this step is not very important in chloroform formation because the formation of chloroform from 2,4dichlorophenol under free chlorination was limited (Fig. S2b). Fiss et al. (2007) also suggest that the majority of the chloroform produced upon chlorination of triclosan was produced via oxidation and ring cleavage of the phenol moiety of triclosan and not from reactions involving the product 2,4-dichlorophenol.

Photolysis only of triclosan can involve oxidation and dechlorination. Thus, a range of photolysis products were detected by Ferrer et al. (2004), including dechlorination at one or more $\mathrm{Cl}$ substituents of triclosan, hydroxylation on the rings, and ether bond cleavage. Antonaraki et al. (2002) reported that UV photolysis and $\mathrm{UV} / \mathrm{H}_{2} \mathrm{O}_{2}$ treatment of chlorophenols involve both dechlorination and hydroxylation, leading to a range of (chlorinated) hydroquinone, quinone, and resorcinol types of structures.

Based on our experimental results, the possible reaction schemes of chloroform formation from triclosan under combined $\mathrm{UV} /$ chlorine conditions are proposed in Fig. $6 \mathrm{~b}$. When chlorine is combined with UV, chlorination, dechlorination and hydroxylation all possibly occurred to triclosan at the beginning of the reaction. Due to the UV photolysis, ring hydroxylation and ether bond cleavage would be more in the combined UV/chlorine system compared to chlorination only. As a result, a series of chlorophenols and (chlorinated) resorcinol types of structures were generated. Because resorcinol types of structures are known to have fast formation rates and high yields of chloroform (Gallard and von Gunten, 2002), the chloroform formation was enhanced in the combined UV/chlorine comparing to chlorination only. The combined UV/chlorine might also facilitate the ring cleavage to chlorinated ketone intermediates that would eventually release chloroform by hydrolysis.

Under the combined $\mathrm{UV} /$ chlorine with relatively high initial chlorine dose (e.g., FAC: $\mathrm{T}=5: 1$ ), the chlorinated triclosan intermediates played the most important role in the formation of chloroform. Fig. 4c shows that the first chlorination alone for $5 \mathrm{~min}$ and post combined UV/chorine generated the maximum chloroform concentrations of $3.02 \mu \mathrm{M}$, which was higher than the maximum chloroform concentrations of $1.76 \mu \mathrm{M}$ under combined UV/chlorine and the initial FAC:T $=5: 1$ (Fig. 2). This result suggests the chlorinated triclosan intermediates, especially the 4,6-Cl-triclosan, were very important intermediates for chloroform formation. On the other hand, the initial UV irradiation alone for $5 \mathrm{~min}$ and post combined UV/chlorine generated the maximum chloroform concentrations of $1.45 \mu \mathrm{M}$ (Fig. 5d). Fig. 5d also shows that after UV irradiation alone for $5 \mathrm{~min}$, a small difference of chloroform formation was observed between the subsequent chlorination 
( a )

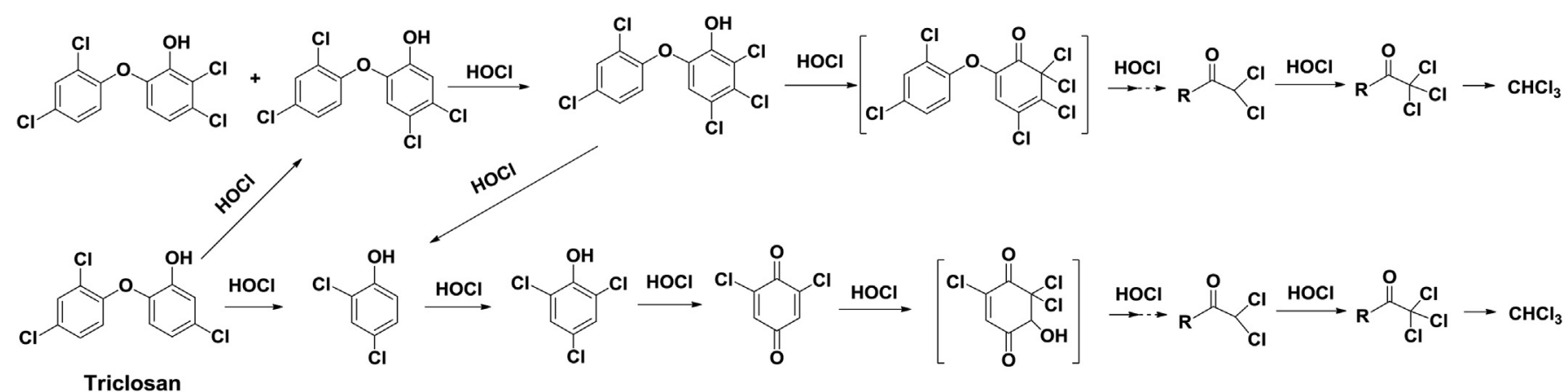

(b)

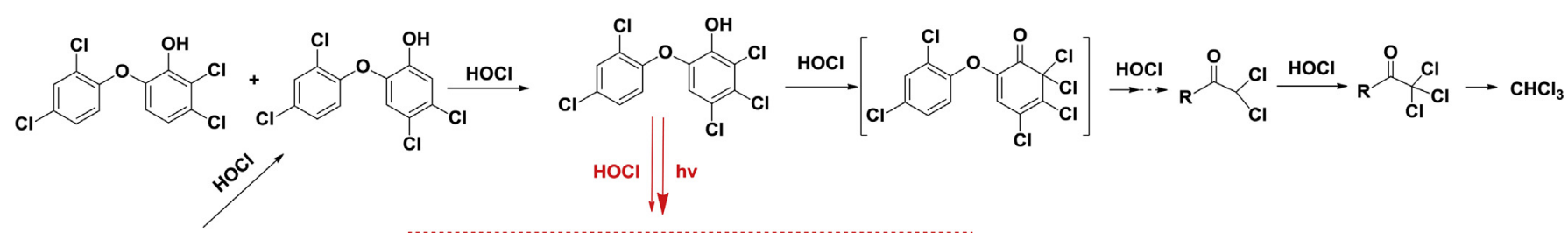

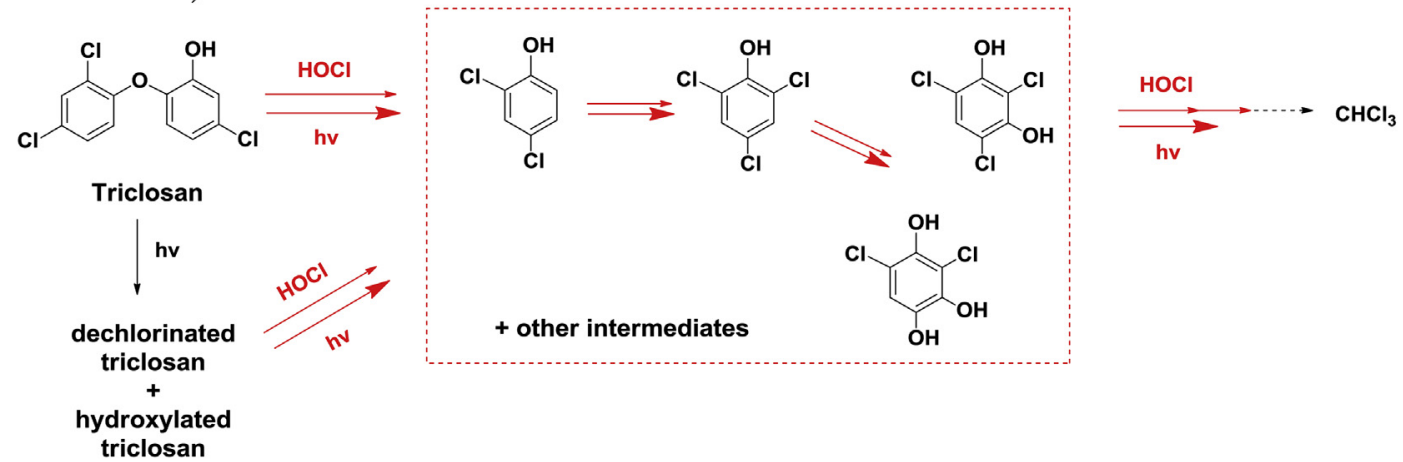

Fig. 6. Proposed degradation schemes of triclosan and formation of chloroform under (a) chlorination alone and (b) combined UV/chlorine conditions.

and the subsequent combined UV/chlorine. These results may indicate that the products from the UV photolysis of triclosan (e.g., dechlorinated/hydroxylated triclosan) were not important intermediates for enhancing the chloroform formation under the combined UV/chlorine and the initial FAC:T $=5: 1$. Although chlorine decayed more under UV, there was still excess chlorine remained in the solution at FAC:T $=5: 1$. Fig. $3 \mathrm{c}$ shows the concentration of dechlorinated triclosan was decreasing along with the increase of the initial FAC concentration. Thus, the dechlorination is likely to be compensated (or reversed) by reaction with chlorine. Fig. 3d shows that the combined UV/chlorine enhanced the formation of 2,4-dichlorophenol in the initial $2 \mathrm{~min}$ and 2,4dichlorophenol maintained at a certain amount within $10 \mathrm{~min}$, implying that the UV irradiation may promote the ether bond cleavage of triclosan and chlorinated triclosan intermediates. 2,4Dichlorophenol intermediate is not a productive chloroform precursors under chlorination only. However, 2,4-dichlorophenol can be quickly further transformed under combined UV/chlorine system to other structures (possibly to 2,4,6-trichloropheol and hydroxylation products). As a result, a series of (chlorinated) resorcinol types of structures were generated, which are much more conducive for chloroform formation. Fig. S2 and Fig. 2 show the similar yields of chloroform formation from 2,4-dichlorophenol and triclosan under combined UV/chlorine and the initial FAC:T $=5: 1$. Hence, 2,4-dichlorophenol might act as an important intermediate in the process of transformation of other chloroform formation precursors (e.g., chlorinated triclosan) to chloroform.

In the combined UV/chlorine process, absorption of UV photons causes the free chlorine species (i.e., $\mathrm{HOCl}$ and $\mathrm{OCl}^{-}$) to generate several radicals such as $\bullet \mathrm{OH}$ and $\bullet \mathrm{Cl}$. Meanwhile, $\mathrm{HOCl}$ and $\mathrm{OCl}^{-}$ can also react with $\bullet \mathrm{OH}$ with reaction rate constants of $8.46 \times 10^{4}$ and $9.0 \times 10^{9} \mathrm{M}^{-1} \mathrm{~s}^{-1}$, respectively (Wang et al., 2012).

$$
\begin{aligned}
& \mathrm{HOCl} \leftrightarrow \mathrm{H}^{+}+\mathrm{OCl}^{-} \quad\left(p K_{a}=7.5\right) \\
& \mathrm{HOCl}+\mathrm{UVphotons} \rightarrow \bullet \mathrm{OH}+\bullet \mathrm{Cl} \\
& \mathrm{OCl}^{-}+\mathrm{UV} \text { photons } \rightarrow \bullet \mathrm{O}^{-}+\bullet \mathrm{Cl} \\
& \bullet \mathrm{O}^{-}+\mathrm{H}_{2} \mathrm{O} \rightarrow \bullet \mathrm{OH}+\mathrm{OH}^{-} \\
& \bullet \mathrm{OH}+\mathrm{HOCl} \rightarrow \mathrm{H}_{2} \mathrm{O}+\mathrm{ClO} \bullet \quad\left(k=8.46 \times 10^{4} \mathrm{M}^{-1} \mathrm{~s}^{-1}\right) \\
& \bullet \mathrm{OH}+\mathrm{OCl}^{-} \rightarrow \mathrm{OH}^{-}+\mathrm{ClO} \bullet \quad\left(k=9.0 \times 10^{9} \mathrm{M}^{-1} \mathrm{~s}^{-1}\right)
\end{aligned}
$$

While the radicals (i.e., $\bullet \mathrm{OH}$ and $\bullet \mathrm{Cl}$ ) may potentially promote triclosan degradation, results show that their impact was negligible (Fig. 2). With regard to the fate of intermediates, chlorinated triclosan intermediates degraded completely within the first 5 min under combined UV/chlorine and the difference between with and without TBA was small (Fig. 3a, b). Thus, the influence of radicals on 
the degradation of chlorinated triclosan intermediates was modest, and these intermediates likely decayed via direct photolysis primarily. Fig. 3d shows that more 2,4-dichlorophenol was generated when radicals were scavenged but the difference was not large, implying radicals had a modest effect in the breakdown of 2,4dichlorophenol. The radicals also showed negligible effect on the dechlorinated triclosan (Fig. 3c). Rather, the dechlorinated triclosan likely decayed by reaction with chlorine or further direct photolysis (Fig. 5c). Fig. 2 shows that removing radicals could lead to a higher level of chloroform after 4-5 min in reaction. This suggests that radicals might react with some of the intermediate compounds and turn them into structures that were less likely to yield chloroform. By comparing the results shown in Fig. $4 c$ and Fig. $5 d$, it can be deduced that the radicals might mainly hinder the transformation of triclosan's photolysis products to chloroform and the direct photolysis might play an important role in the transformation of chloroform formation precursors in the combined UV/chlorine treatment. On the other hand, the quenching of $\bullet \mathrm{OH}$ might decrease the consumption of free chlorine (particularly $\mathrm{OCl}^{-}$) in the system, resulting in a slightly higher chlorine abundance than without quenching.

\section{Conclusions}

The combined UV/chlorine can notably enhance the chloroform formation from triclosan comparing to chlorination alone. In the combined system, both chlorinated triclosan intermediates and photolysis products of triclosan were generated, while the chlorinated triclosan intermediates may act as more important precursors of chloroform, especially at high initial chlorine dose. The UV photolysis played an important role in the decay of triclosan and its chlorinated/dechlorinated intermediates by promoting ring hydroxylation and cleavage. As a consequence, the intermediates may transform to a series of structures (e.g., (chlorinated) resorcinol types) which are more conducive for chloroform formation. The results of this work indicate that the combined UV/chlorine may promote the formation of toxic chlorinated by-products from organic pollutants, which is necessary to be considered in the combined application of chlorine and UV or other systems of coexposure of UV irradiation and free chlorine.

\section{Supplementary material}

Supplementary data related to this article can be found online.

\section{Acknowledgments}

Funding support from the Chinese Scholarship Council for W. Ben is gratefully acknowledged.

This work was also financially supported by the National Natural Science Foundation of China (No. 21107127).

\section{Appendix A. Supplementary data}

Supplementary data related to this article can be found at http:// dx.doi.org/10.1016/j.chemosphere.2015.12.071.

\section{References}

twentieth ed. American Public Health Association, Washington, D.C.

Antonaraki, S., Androulaki, E., Dimotikali, D., Hiskia, A., Papaconstantinou, E., 2002. Photolytic degradation of all chlorophenols with polyoxometalates and $\mathrm{H}_{2} \mathrm{O}_{2}$. J. Photochem. Photobiol. A: Chem. 148 (1-3), 191-197.

Arnold, W.A., Bolton, J., von Gunten, U., Hofstetter, T.B., 2008. Evaluation of functional groups responsible for chloroform during water chlorination using compound specific isotope analysis. Environ. Sci. Technol. 42 (21), 7778-7785.

Buth, J.M., Ross, M.R., McNeill, K., Arnold, W.A., 2011. Removal and formation of chlorinated triclosan derivatives in wastewater treatment plants using chlorine and UV disinfection. Chemosphere 85 (2), 284-289.

Deng, L., Huang, C.H., Wang, Y.L., 2014. Effects of combined UV and chlorine treatment on the formation of trichloronitromethane from amine precursors. Environ. Sci. Technol. 48 (5), 2697-2706.

Fang, J., Fu, Y., Shang, C., 2014. The roles of reactive species in micro-pollutant degradation in the UV/free chlorine system. Environ. Sci. Technol. 48 (3) 1859-1868.

Feng, Y., Smith, D.W., Bolton, J.R., 2007. Photolysis of aqueous free chlorine species $\left(\mathrm{HOCl}\right.$ and $\left.\mathrm{OCl}^{-}\right)$with $254 \mathrm{~nm}$ ultraviolet light. J. Environ. Eng. Sci. 6 (3), 277-284.

Ferrer, I., Mezcua, M., Gómez, M.J., Thurman, E.M., Agüera, A., Hernando, M.D., Fernández-Alba, A.R., 2004. Liquid chromatography/time-of-flight mass spectrometric analyses for the elucidation of the photodegradation products of triclosan in wastewater samples. Rapid Commun. Mass Sp. 18 (4), 443-450.

Fiss, E.M., Rule, K.L., Vikesland, P.J., 2007. Formation of chloroform and other chlorinated byproducts by chlorination of triclosan-containing antibacterial products. Environ. Sci. Technol. 41 (7), 2387-2394.

Gallard, H., von Gunten, U., 2002. Chlorination of phenols: Kinetics and formation of chloroform. Environ. Sci. Technol. 36 (5), 884-890.

Heidler, J., Halden, R.U., 2007. Mass balance assessment of triclosan removal during conventional sewage treatment. Chemosphere 66, 362-369.

Jin, J., El-Din, M.G., Bolton, J.R., 2011. Assessment of the UV/Chlorine process as an advanced oxidation process. Water Res. 45 (4), 1890-1896.

Kemsley, J., 2014. Triclosan under the microscope. Chem. Eng. News 92 (25), 10-13.

Kolpin, D.W., Furlong, E.T., Meyer, M.T., Thurman, E.M., Zaugg, S.D., Barber, L.B. Buxton, H.T., 2002. Pharmaceuticals, hormones, and other organic wastewater contaminants in U.S. streams, 1999-2000: a national reconnaissance. Environ. Sci. Technol. 36 (6), 1202-1211.

Lindström, A., Buerge, I.J., Poiger, T., Bergqvist, P., Müller, M.D., Buser, H., 2002. Occurrence and environmental behavior of the bactericide triclosan and its methyl derivative in surface waters and in wastewater. Environ. Sci. Technol. 36 (11), 2322-2329.

Loraine, G.A., Pettigrove, M.E., 2006. Seasonal variations in concentrations of pharmaceuticals and personal care products in drinking water and reclaimed wastewater in Southern California, Environ. Sci. Technol 40 (3), 687-695.

Nowell, L.H., Hoigné, J., 1992. Photolysis of aqueous chlorine at sunlight and ultraviolet wavelengths-II. Hydroxyl radical production. Water Res. 26 (5) 599-605.

Rule, K.L., Ebbett, V.R., Vikesland, P.J., 2005. Formation of chloroform and chlorinated organics by free-chlorine-mediated oxidation of triclosan. Environ. Sci. Technol. 39 (9), 3176-3185.

Sichel, C. Garcia, C., Andre, K., 2011. Feasibility studies: UV/chlorine advanced oxidation treatment for the removal of emerging contaminants. Water Res. 45 (19), 6371-6380.

Singer, H., Müller, S., Tixier, C., Pillonel, L., 2002. Triclosan: occurrence and fate of a widely used biocide in the aquatic environment: field measurements in wastewater treatment plants, surface waters, and lake sediments. Environ. Sci. Technol. 36 (23), 4998-5004.

Soltermann, F., Lee, M., Canonica, S, von Gunten, U, 2013. Enhanced N-nitrosamine formation in pool water by UV irradiation of chlorinated secondary amines in the presence of monochloramine. Water Res. 47 (1), 79-90.

Son, H.S., Choi, S.B., Zoh, K.D., Khan, E., 2007. Effects of ultraviolet intensity and wavelength on the photolysis of triclosan. Water Sci. Technol. 155 (1-2) 209-216.

Wait, I.W., 2008. Multiple-Barrier disinfection by chlorination and UV irradiation for desalinated drinking waters: chlorine photolysis and accelerated lamp-sleeve fouling effects. Water Environ. Res. 80 (11), 2183-2188.

Wang, D., Bloton, J.R., Hofmann, R., 2012. Medium pressure UV combined with chlorine advanced oxidation for trichloroethylene destruction in a model water. Water Res, 46 (15), 4677-4686.

Weng, S., Li, J., Blatcheley, E.R., 2012. Effects of $U_{254}$ irradiation on residual chlorine and DBPs in chlorination of model organic-N precursors in swimming pools. Water Res. 46 (8), 2674-2682.

Ying, G., Kookana, R.S., 2007. Triclosan in wastewaters and biosolids from Australian wastewater treatment plants. Environ. Int. 33 (2), 199-205.

Zhao, Q., Shang, C., Zhang, X., Ding, G., Yang, X., 2011. Formation of halogenated organic byproducts during medium-pressure UV and chlorine coexposure of model compounds. Water Res. 45 (19), 6545-6554. 